De la elaboración de cada etapa se desprenden unas conclusiones, pero a la vez se abren nuevos campos de estudio. El trabajo realizado marca unas líneas de actuación que nos proponemos desarrollar en un futuro plan de investigación, con el que reafimar las conclusiones y a través del cual llegar a una implantación efectiva del Analisis Numerico en el Bachillerato, o en los cursos de Secundaria del próximo plan de estudios.

ANÁLISIS Y EVALUACIÓN COGNITIVA DE LA SITUACIÓN EDU. CATIVA: UNA APROXIMACIÓN EMPIRICA A LA METAEVALUACIÓN DEL PROFESOR

\section{(Tesis Doctoral)}

Autor: Ángel Latorre Latorre.

Director: Dr. D. Francisco Rivas Martinez.

Departamento de Psicología Evolutivay de la Educación.

Presentado en febrero de 1989 en la Universitat de València.

La investigación realizada, podría ubicarse en el punto de confluencia entre la Psicología del Procesamiento de la In- formación, la Psicologia de la Instrucción y la Psicología Educativa. Por ser la Psicología de la Instrucción la que se centra en los conocjmientos básicos, concretos y específicos, y en la forma de estructurarlos en el sujeto, es quizás la que proporciona el marco más apropiado para estudiar las representaciones cognitivas.

El objetivo de la investigación pretende captar la representación cognitiva que de la Situación Educativa han estructurado los profesores de ciencias, mediante un análisis y evaluación cognitiva de la Situación Instrucional, desde una perspectiva experimental, centrada en la metaevaluación que el profesor de Ciencias realiza sobre una Situación de enseñanza/aprendizaje concreta, desarrollada en su clase, en condiciones de total normalidad escolar, al aplicar un diseño de instrucción realizado con una unidad específica de contenido.

Lafundamentación teórica del trabajo se cimenta en: a) Teorías Representacionales de la cognición. b) Implicaciones mutuas entre conocimiento y acción. c) Conocimientos profesionales de los profesores.

En la parte teórica, a través de una doble aproximación mediante un analisis macrosistémico y microsistémico, llegamos a diferenciar los tres tipos de variables incidentes en la Situación Educativa: intrapersonales y situacionales. Seleccionando de entre todas las variables la representación cognitiva que el profesor establece sobre la Situación Instruccional tomada como centro de la investigación.

En la parte experimental se plantea un análisis de la Situación Educativa desde un punto de vista empírico, empleando cuestionario, tabla de calificación y dominio de objetivos de aprendizaje, rejilla (grid) y diseños de instrucción, podemos de esta forma controlar las variables de profesor y estudiante a través de diferentes niveles educativos (Preescolar, C. Inicial, C. Medio y C. Superior).

Los resultados obtenidos confirman, desde el punto de vista experimental, la incidencia que sobre el proceso de ensenanza/aprendizaje ejercen las percepciones del profesor. Se derecta la existencia de diferencias significativas en la estructuración y representación cognitiva que los profesores poseen sobre la Situacion Educativa, en función de diversas variables, siendo las más abundantes y con mayor grado de significación, las referidas a la experiencia docente (expertise). Se evidencia con ello la importancia que posee la práctica en la estructuración de esquemas cognitivos que permiten al profesor experto prever los eventos de los procesos de enseñanza/aprendizaje con un mayor grado de eficacia.

\title{
CENTROS DE FORMACIÓN DEL PROFESORADO
}

\section{POST GRADO EN DOCENCIA DE LAQUIMICA EN LA UNIVERSIDAD PEDAGógICA NACIONAL (CO- LOMBIA)}

La Universidad Pedagógica Nacional de Bogotá (Colombia) con el auspicio del programa estatal para el desarrollo de la capacidad de investigación ofrece desde 1987 un programa de Magister en Docencia de la Química, cuyos objetivos básicos son:

Desarrollar actitudes científicas e investigativas para la docencia de la química en sus participantes.

Contribuir, mediante el desarrollo de la investigación didáctica al conocimiento de la metodología de la enseñanza de la química en el país y proponer aiternativas de solución a los problemas allí planteados.
Formar personal cualificado para asumir las responsabilidades pertinentes a la investigación y docencia de la química.

Formar pensadores de la química, conocedores de las estructuras conceptuales de la misma y su metodología de producción, capaces no solamente de utilizarlos para generar nuevos conocimientos, sino también como medio de forjar actitudes cientfficas.

La estructura curricular del postgrado contempla una fundamentación teórica, una fundamentación técnica, la investigación didáctica y actividades complementarias.

La fundamentación teórica se entiende como el conjunto de actividades mediante las cuales el estudiante, debidamente orientado por el profesor, busca estructuras básicas dentro de las diferen- tes áreas de la química, profundiza dentro de ellas y adquiere una visión global de las mismas.

La fundamentación técnica busca inducir formativamente al estudiante en los procesos de adquisición de destrezas para el manejo seguro y eficiente de los instrumentos de laboratorio, la obtención e interpretación de resultados y la obtención de generalizaciones.

La estructura del programa permite, además, el desarrollo de conferencias, asistencia a eventos científicos y cursos cortos.

El aspecto fundamental del postgrado es la investigación didéctica, o sea, ia problemática alrededor de Ia cual gira el proceso de enseñanza-aprendizaje de la química. Actualmente se desarrollan trabajos en dos líneas de investigación: 
"problemas pedagógicos y didácticos en el aprendizaje de conceptos en química" y "contribución a la enseñanza de la química, a través de la caracterización de ambientes naturales colombianos".

La primera línea parte de Ia existencia de preconceptos, ideas intuitivas o esquemas alternativos elaborados por el individuo en su experiencia diaria, con lo cual explica e interpreta los fenomenos que ocurren a su alrededor. Centra su interés en las características y en Ios efectos interferentes que estos esquemas conceptuales puedan tener en el aprendizaje de los conocimientos científicos y de las implicaciones de este hecho en el diseño de estrategias pedagógicas que pretendan mejorar las metodologías tradicionales utilizadas en la enseñanza de la química.

La segunda línea, a partir del análisis de la bibliograffa relacionada con la enseñanza de las ciencias naturales en Colombia, así como también de la experiencia diaria en el desarrollo de la profesión docente, concluye que la enseñanza es artificial, ya que se desconocen los procesos de producción del conocimiento científico, las circunstancias que lo rodean y se carece de claridad acerca de cuáles son los contenidos de la ciencia y el papel que debe jugar en una sociedad. La línea se orienta hacia la búsqueda de mecanismos para la "desartificialización" y conversión de esta situación en un verdadero proceso de desarrollo del pensamiento y de la actitud científica de Ios estudiantes, haciendo énfasis en la metodología de producción del conocimiento científico y en su naturaleza pedagógica y didáctica, mediante el estudio sistemático de un ambiente natural.

El Postgrado en Docencia de la química está dirigido a todos los profesionales de la química, preferiblemente a aquéllos dedicados a la docencia universitaria para la formación de profesores. Los aspirantes a participar en el programa deben: enviar hoja de vida (Curriculum Vitae), diligenciar el formulario de inscripción y presentar calificaciones del pregrado, acreditar título profesional universitario, presentar certificaciones de experiencia docente, de investigación o administrativas en el área de la química en instituciones de educación, demostrar disponibilidad de tiempo para dedicarse exclusivamente a la realización del programa, demostrar capacidad para comprender información científtca en ingles, presentarse a las pruebas de seleccion y realizar una entrevista personal.

Dada la problemática común que afrontan los países latinoamericanos en cuanto a la enseñanza-aprendizaje de la química, el programa está abierto para estudiantes extranjeros que a través de instituciones internacionales ode sus propios países, manifiesten su deseo de ingresar al programa y que a juicio del comité de postgrado cumplan los requisitos establecidos para tos estudiantes nacionales.

Los interesados en participar o en intercambiar información pueden dirigirse a:

Universidad Pedagógica Nacional

Departamento de química

Calle 72 N $11-86$

Bogotá, Colombia, Sur América

Luts E. Salcedo

\section{NOTICIAS}

\section{PREMIOINTERNACIONAL POR UN FUTURO CREATIVO}

El Comité Intemacional parala Promoción de la Investigación Educativa Avanzada (C.A.P.I.R.E.) persigue con este premio realzar las investigaciones y experimentaciones educativas que tiendan a una profunda renovación de los sistemas educativos contemporáneos.

En este sentido los trabajos que se presenten a este premio deben corresponder a una de las siguientes categorías:

a) Trabajos teóricos sobre aprendizaje creativo;

b) Experiencias e innovación educativa;

c) Periodismo científico;

d) Museos y ferias de didáctica interactiva.

Los participantes al "Premio por un Futuro Creativo" tienen que presentar sus trabajos en forma "audiovisual" (vídeo-cassette VHS con una duración de 30 minutos). Los audiovisuales premiados se presentarán a través del proyecto "Educacion" del satélite Olimpus en un programa de la RAI-TV italiana.

Los trabajos pueden remitirse a:

Paolo Manzelli (Secretario del CAPIRE)

Laboratorio Ricerca Educativa,

Via Maragliano 77, (Universitádi Firenze). Firenze (Italia)
La primera edicion del "Premio para un futuro creativo" tendrá lugar el 30 de abril de 1990 en el Museo d'Arte Contemporanea 'L'PECCI' di Prato (Firenze).

\section{HISTORY TEACHING PHYSICS}

\section{St.John's College, Cambridge, Great Bri-} tain.

28-30 August 1990

The Interdivisional Group on the History of Physics of the European Physical Society in conjunction with the Institute of Physics and the Cambridge Physics History Group, is holding a three day meeting designed to support teachers and educationalists interested in the use of the history of science in the teaching of physics. This is an international meeting, and is one of a series previously held in Pavia, Munich and Paris, and represents a major opportunity for those interested in the pedagogical applications of the history of science to meet colleagues with similar interests from all major European countries.

Sessions will include:

- History of science in the physics curriculum

- Recent trends in the history, philosophy and sociology of science
- Intellectual development and the history of physics

- ParaileI workshop sessions on the above themes and other topics (currently proposed: the use of historial experiments in science teaching, current trends in the historiography of physics, physics and gender).

A certificate will be awarded to delegates attending throughout the three days.

A full programme of speakers and workshops will be announced in May 1990. Anyone wishing to receive this announcement and a booking form should complete and return the section below to: Ms Becky Parker, The Institute of Physics, 47 Belgrave Square, London, SWIX 8QX, Great Britain.

\section{History Teaching Physics}

Name

Institution

Address

Telephone Number .

Fax Number 